\section{DISYUNTIVAS ONTOEPISTÉMICAS DE LOS SABERES HACERES DOCENTES}

ONTOEPISTÉMIC DILEMMAS OF TEACHING KNOWLEDGE AND TASKS

Ana Méndez de Garagozzo* Morela Arráez Belly **

UPEL-IPB

Recibido: 08-05-07

\section{RESUMEN}

Este artículo aborda la comprensión integradora del saber hacer docente, como un proceso de deconstrucción y reconstrucción desde la dualidad sujeto-objeto en transformación permanente durante la labor educativa. La postura epistémico teórica se fundamenta en una cosmovisión articulada al enfoque sociocrítico para analizar los fundamentos ideológicos de las prácticas, reconstructora de las formas de conocer y relacionarse para conocer, según Bacherlad (1985), Schon (1992), segina (1986) y Morin (1999). La (1992), Habermas (1986) y Morin (1999). La revisión documental permitió, desde la hermenéutica, la exploración de diseños, métodos y estrategias para la comprensión y valoración de la información, lo que generó un sentido de profundidad en la cientificidad de conocimiento. Concluyendo que la docencia universitaria es una práctica tensionada, emerge una subjetividad e intersubjetividades motivadas al cambio, en la consecución de prácticas liberadoras que provoquen rupturas y conformen un pensamiento que se realice en la cotidianidad, para redimensionar la realidad y la praxis educativa.

\section{INTRODUCCIÓN}

1 Este trabajo está adscrito a la Línea de Investigación: Saber Hacer Docente de la UPEL-IPB

* Ingeniera Electrónica, Magíster en Matemática, Doctora en Ciencias de la Educación Coordinadora de la línea Saber Hacer Docente y del Programa Electricidad Industrial de la UPEL-IPB. Profesora agregado a dedicación exclusiva de la UPEL-IPB. Mención Honorífica y Premio Iberoamericano de Creatividad e Innovación 2005 y 2007, México. Miembro del Programa de Promoción al Investigador (PPI). e-mail: anamendezg@hotmail.com

** Profesora egresada de la UPEL, Instituto Pedagógico "Luis Beltrán Prieto Figueroa" en Castellano y Literatura, Magíster en Administración Educacional y Doctora en Ciencias de la Educación egresada de la Universidad "Santa María”.Investigador en las Líneas "Lingüística Aplicada a la Enseñanza de la Lengua Materna" y "Comportamiento Organizacional". Miembro de Programa de Promoción al Investigador (PPI) e-mail: morelarraez@gmail.com
En una sociedad caracterizada por ritmos de cambio muy diversos, emergen retos en la formación de los profesionales, sobre todo para los formadores de formadores, quienes deben ser capaces de navegar en una era caracterizada por la incertidumbre y la complejidad social, económica, política y educativa; cuya mayor certeza es la permanencia que muestran los cambios de la realidad social. Por ello las organizaciones educativas efectivas requieren estar preparadas para responder a una multiplicidad de eventualidades y ser lo bastante flexibles para implementar nuevos direccionamientos, que conciban respuestas de calidad a las necesidades vinculadas a su entorno en un devenir incierto.

En tal sentido, el ritmo evolutivo actual estima que los docentes en la sociedad posmoderna comiencen a repensarse, para accionar desde las nuevas posturas emergentes, en el entendido que se debaten entre fuerzas de conservación y de cambio, por cuanto el espejismo de sentirse cobijados en la seguridad conservadora de las formas tradicionales, induce a la permanencia de ciertos modos que se amparan en los dogmas, las tradiciones y las costumbres, arraigados en el sentido común, en la vida cotidiana, es decir, la vida que es vivida, posibilitando la continuidad de comportamientos rutinarios, heredados y poco reflexionados críticamente.

Cabe entonces plantear lo señalado por Sinclair (1981) "los métodos empíricos en los que el sujeto debe aceptar la existencia de un vínculo entre los hechos debido a que se le impone dicho plazo, no arrojan progreso alguno; éste se logra cuando el propio sujeto descubre la relación entre los hechos” (p. 35). La postura del autor permite inferir que las modificaciones de los esquemas cognitivos en la construcción del conocimiento, es un proceso continuo, donde los factores intrínsecos y las condiciones del entorno socio-cultural juegan un rol fundamental para el avance creciente de las estructuras intelectuales, así como la autodeterminación de dialogar con la concepción de la realidad percibida, tal como se presenta en los espacios de conciencia, en la ocasión de definir y articular la cognición, es decir, el conocimiento.

Por otra parte, el docente del siglo XXI tiene que enfrentarse a las formas y mecanismos de los desenvolvimientos actuales, caracterizados por un exceso en el consumo, la influencia de los mass media y un olvido creciente, no sólo el olvido de lo consumido y la sed de la novedad, sino el olvido del otro, de su naturaleza y su diversidad, con un materialismo que se sobrepone a la condición humana y a los valores subyacentes en el ejercicio cívicodemocrático. En esta circunstancia, se estima que el mundo de hoy debe entenderse desde una perspectiva histórica, es decir un posicionamiento que convoque a una conciencia histórica, para darle consistencia al momento actual y una dimensión a la vida, sin desconcertarse ante las complejas eventualidades del presente que desubican y tienden a desequilibrar al ser, al propiciar divergencias en los modos de pensar, sentir y actuar. 
En este orden de ideas, cabe destacar que la tecnología irrumpe con la hegemonía socializadora y de transmisión de saberes que tradicionalmente se ha depositado en la escuela, la cual es objeto de críticas y atraviesa por una crisis característica de las instituciones de la modernidad, porque actualmente son los medios de comunicación quienes socializan a los jóvenes, proporcionándoles modelos, estilos de vida y comportamiento, que establecen marcadas diferencias y distancia entre el sistema educativo, el proceso de formación, la experiencia y la sensibilidad de los sujetos, fundada en una racionalidad comunicativa que disocia el modelo escolar y la dinámica que se plantea en la sociedad a través de la tecnología.

Es así como la verdad del saber técnico está sustituyendo a la verdad filosófica, la cual es anterior a la pretendida verdad acabada lógico numérica del paradigma positivista y se está convirtiendo en un simbolismo que no permite la ocupación y preocupación por construir el futuro, sino que promueve la inmediatez de las cosas y de los instrumentos en aras de lo real inmediato, desvirtuando lo real humanizado y su naturaleza constructiva.

Por ello, es necesario posicionarse en el tiempo y en el espacio, convivir con las formas que la vida cotidiana presenta, pero con la conciencia de una historia que se hace presente y desde ella construir el futuro, de tal manera que como seres concretos se participe históricamente, en busca de la constitución del sujeto social, instituido desde las subjetividades y las intersubjetividades en la proyección de un futuro sostenible, para realizar los proyectos, las utopías y las fuerzas que se propongan, como posibilitadores de nuevas realidades.

Al respecto cabe considerar lo planteado por Barbero (2006) cuando señala que "la verdad de la existencia humana no se agota en el saber ligado a la técnica, pues hay otro saber que es el saber-del-ser" (p. 18), desde donde se desoculta la dimensión humana de innovar, pero también reside el peligro de olvidar.

Luego, hay que considerar que los docentes, amparados en el paradigma que irradia el pensamiento consciente, el cual es también subconsciente, como afirma Morin (1999), actúan de acuerdo a una postura ontológica determinística, convertida en una cosmovisión que ha penetrado y se ha perpetuado en las universidades, direccionando no sólo el pensar y el actuar sino también el sentir, convirtiéndose en un estilo dominante.

Por lo tanto, la herencia que la modernidad ha dejado, requiere ser deconstruida para develar lo oculto y poder aclarar sus desaciertos y aciertos, en el entendido que un paradigma que persigue el control, la reproducción, la fragmentación y la reducción, poco puede aportar a un pensamiento emergente, que vislumbre un horizonte cuyo sentido sea construirse colectivamente, con un fin integrador, capaz de propiciar nuevas realidades en forma comprensiva y no controlada, ni controladora, desde una postura humanizada y humanizante del ser, creadora de una conciencia ecológica emparentada con una identidad terrenal.

En este sentido se considera significativo que el docente universitario se interrogue en forma permanente sobre el accionar educativo y social que desarrolla, ya que la falta de preguntas es infinitamente peor que la falta de respuestas, porque no se reflexiona sobre la necesidad de cambio, ni se propone el análisis crítico reconstructivo que le ayude a posicionarse ante la diversidad cultural. Asimismo, debe repensarse y con un estilo innovador trascender su alienación y reconstruir su identidad, reflexionada críticamente, pues según Barabtarlo (1998), su identidad es deficitaria, al ser excluido del proceso del cual es producto y producente, igualmente de la planeación e investigación de la práctica que realiza, por ello requiere definirse como el autor, actor y agente activo de los cambios y las transformaciones de su acción educativa.

En consecuencia la UNESCO, a través de la Declaración Mundial sobre la Educación Superior en el Siglo XXI (1999), propone a la innovación como un criterio pertinente para el desarrollo en las universidades, por consiguiente habría que plantearse ¿Qué pensamiento requiere reconstruir el docente? ¿Acaso este proceso es corolario de la reflexión crítica compartida?, aproximar las respuestas a estas interrogantes permite dilucidar la trascendencia de un pensamiento que se reorganice continuamente para su realización en la cotidianidad, a fin de posibilitar la reconstrucción y el develamiento de los autoentendimientos y las ideologías dominantes, así como la emergencia de una ideología crítica, ubicada no sólo a nivel del discurso sino de la acción articulada con éste, que promueva novedosas formas de comprender y construir los saberes, al mediar el aprendizaje de los estudiantes en contextos concretos, con un aquí y un ahora, para que realicen significativamente las reconstrucciones a las que tienen que arribar.

Aproximar las respuestas a las interrogantes planteadas constituye la intencionalidad de esta investigación documental, donde se pretende a través de la exégesis de diversas teorías, comprender el fundamento y la constitución de las ideologías dominantes en los docentes y discentes del presente siglo, con el propósito de promocionar una ideología crítica que permita la reformulación de los saberes haceres, acorde con una sociedad compleja, abierta y pluralista como la actual.

\section{DISYUNTIVA ONTOEPISTÉMICA DEL DOCENTE EN EL SIGLO XXI}

La disyuntiva ontoepistémica que vivencia el docente del siglo XXI, se expresa en la confrontación y el debate que instaura entre las formas tradicionales de accionar en el aula, en el ámbito educativo, en el social y los nuevos modos de pensar, sentir y actuar, fundados en las cosmovisiones emergentes. 
Estos modos tradicionales, amparados en posturas positivistas que devienen del realismo y su modelo epistémico, el empirismo, lo inducen a reproducir un saber hacer heredado, poco reflexionado, constituido y direccionado en su esencia, por ideologías dominantes, convertidas en sentido común a través de las costumbres y las tradiciones, lo cual se comporta como un obstáculo epistemológico a la construcción del conocimiento, al desarrollo personal y profesional del educador, a la promoción de nuevas realidades educativas, según lo expresa Bachelard (1985), pues amparados en esta forma de vida académica la visión paradigmática positivista los dirige a comprometerse y realizar la acción docente de acuerdo a modelos pedagógicos tradicionales, academicistas o conductistas.

La concepción y enfoque epistemológico de la educación en la perspectiva del Estado queda reflejado en dos saberes: saber y saber hacer para ser competentes. Lo que supone que, el saber hacer en contexto se refiere al conjunto de acciones que se realizan en un ámbito específico, cumpliendo con las exigencias que conlleva a una persona a producir un trabajo o resolver un problema del entorno. En esta investigación, los saberes haceres se consideran fenómenos complejos, inmersos en los saberes de la práctica, pues devienen de procesos reflexivos y sistemáticos que consiguen su completud y síntesis en la acción educativa desarrollada en contextos donde se articulan dialógicamente la teoría y la práctica, a través de ejercicios metacognitivos y sociofectivos.

Manterola (s/fecha) afirma que si la postura, el enfoque epistémico, se fundamenta en el positivismo, se tiene una concepción del conocimiento como algo acabado, autoconstituido, cuya verdad tiene un máximo porcentaje de certeza y es determinística, por consiguiente los estudiantes y el docente tienen que repetir estos conocimientos, entendidos como información y acumulación, debe memorizarlos, porque representan una verdad acabada que el docente posee en su totalidad, por ello es el centro del proceso de enseñanza y aprendizaje, es directivo y tiende a ser autoritario.

Esta es una realidad que se describe con cierto temor, pero es el regreso al hilo, como diría el poeta Andrés Eloy Blanco, que entreteje la trama que representa nuestro actual modelo educativo. Por lo tanto, de acuerdo a los postulados de las nuevas visiones y posturas, se requiere trascender estas formas tradicionales, que consideran a un docente rutinario y unos estudiantes pasivos, quienes poco reflexionan críticamente sobre sus acciones, sus pensamientos y sentires. Igualmente, es necesario develar la tradicional inclinación de la universidad hacia el positivismo, pues ella puede convertirse en un obstáculo pedagógico y representar un cuello de botella donde todos terminarán siendo empiristas, como afirman Porlán y Rivero (1998).

En consecuencia, se estima solventar esta naturaleza reproductora y acrítica, para dar paso a un docente activo, dinámico, comprometido con el estudiante, con sus pares, con la universidad y consigo mismo. Sobre todo con lo relacionado a la construcción pertinente del conocimiento, capaz de dar cuenta de novedosos estilos cognitivos, innovador y democrático, sensibilizado ante las necesidades de sus alumnos, en cuanto a lo disciplinar, lo afectivo, lo social y cultural, a través de una racionalidad comunicativa y afectiva, potenciadora de las cualidades creativas del estudiante, para que conjugue lo afectivo y lo cognitivo en la personalidad, como lo refieren González y Mitján (1989), constituido a través de un proyecto ético de vida, que posibilite un desarrollo autónomo del estudiante para emprender desaprender y aprender en forma permanente.

En este despertar, que introduce un nuevo y pertinente posicionamiento, el docente requiere realizar reflexiones conscientes en forma individual y colectiva, con fines articuladores, pues un obstáculo pedagógico, que se opone al desarrollo del docente y la constitución de una praxis liberadora es la escinción entre la teoría y la práctica. Luego, el docente debe comprometerse en acciones creativas e innovadoras que estimen el establecimiento de puentes vinculadores entre la teoría y la práctica, fundamentados en la reflexión crítica reconstructiva, como elemento articulador de ellas, según estima Shön (1992), pues la acción intenta poner en práctica la eficiencia humana y la reflexión determina cómo aprenden.

En estos modos se compromete una inmersión en el enfoque epistémico relativo al sociocrítico o introspectivo vivencial, donde el conocimiento se genera a partir de la comprensión de los simbolismos socioculturales y la comprensión de las subjetividades que comparte un grupo, así como la reflexión sobre las experiencias vivenciadas, todas las cuales se requieren para operar exitosamente en la cultura actual, manejando eficiente y responsablemente las nuevas tecnologías.

Es por ello que la teoría social crítica pretende integrar la teoría y la práctica en forma dialéctica, mediante la reflexión, el conocimiento y la acción política. Se concibe la teoría y la práctica como producto de construcciones sociales en contextos reales y concretos, soportadas por una ideología, como afirma Giroux (1990), quien establece que la dimensión ideológica que conforma el pensamiento reflexivo crítico, enuncia los valores históricos y sociales que participan en la construcción del conocimiento, en las relaciones sociales y en las prácticas.

Desde esta postura se encaran los cambios con una naturaleza dinámica y crítica, consecuente con formas emancipadas de ser y de actuar, así como estrategias de participación que procuran la construcción de lo real humanizado, en franco alejamiento de lo real inmediato y su interés reproductor, por lo que no se legitiman espacios históricamente determinados, sino que se trabaja en la construcción social de una cultura que reconozca al otro, desde su diversidad, respetando sus ideas y analizando las diferencia para considerarlas y comprenderlas. 
Cabe destacar que el docente desde esta postura reflexiva e integradora, articulará los saberes, fundado en los principios organizadores de un pensamiento complejo como propone Morin (ob.cit.), desarrollando la capacidad de instaurarlos en un contexto para su realización en la cotidianidad, a través de una racionalidad dinámica, capaz de volver sobre sí, con la finalidad de reorganizar su pensamiento y elaborar las nuevas construcciones con sentido humanista, develándose como el capital intelectual de la universidad, al articular los conocimientos con y en la creación de la cultura

De este modo, el conocimiento que adquieren los profesores en la formación no se relaciona de una manera implícita con la práctica, para que éste se transforme en conocimiento práctico, requiere la implementación y el uso de estrategias metacognitivas, entendida la metacognición como un tipo de reflexión orientada a un saber hacer, para mejorar lo que se hace, que conlleve cambios conceptuales, actitudinales y procedimentales. Por consiguiente, no se refiere a una concepción mecanicista, sino a un proceso de toma de conciencia que confronte el pensamiento y la acción, los articule e impregne con los sentimientos.

Es así que el conocimiento se considera un elemento muy valioso para cualquier institución, organización, grupo o persona. La información, el conocimiento y el aprendizaje son aspectos consustanciales con las personas y las inteligencias son las claves que explican el sentido de las transformaciones sociales, científicas y tecnológicas.

Por consiguiente, el desarrollo de la inteligencia emocional propicia que se estructuren las acciones en la intención social, con la inteligencia racional reconstruirá los saberes con capacidad para contextualizarlos, con la inteligencia espiritual propondrá nuevos sentidos, sobre todo, un sentido ético de la vida y propiciará las evoluciones desde su naturaleza creativa; todo esto aunado a la constitución del sujeto epistémico, quien objetivará los objetos del conocimiento y articulará los saberes, para instituirse como el sujeto orgánico transformador al interrelacionarse con los demás, con sentido compartido y colaborativo, a fin de develarse como el capital intelectual, académico y social de la universidad.

En este sentido vivenciará el saber hacer, a través de nuevas formas de ser y convivir, con una renovada visión del conocer, en beneficio de los actores del proceso de enseñanza y aprendizaje, manifestándose en la sociedad al colaborar de manera significativa, con aportes dirigidos a mejorar la calidad de vida de sus miembros, a través de otorgar un nuevo significado a su acción educativa.

De tal manera que su acción será consustancial con una actitud que contribuye a sustentar y promover la consideración del criterio de pertinencia social y calidad, para impulsar el capital intelectual en las universidades y estimado, asimismo, en los centros de formación superior del siglo XXI, con el propósito de convertirse en ámbitos de generación del saber, polos de desarrollo, de transferencias significativas y del resguardo de los valores, trascendiendo su herencia colonial domesticadora en pro de la satisfacción de las necesidades básicas de aprendizaje, determinadas por las exigencias y desafíos del nuevo siglo.

\section{CRITERIOS PARA LA CONSTITUCIÓN DEL DOCENTE EN EL SIGLO XXI}

Los criterios principales señalados en la Declaración Mundial para la Educación Superior del Siglo XXI, Tünnermam (ob.cit.), referidos a la calidad, la pertinencia social y la internacionalización, son piedras angulares que fundamentan el pensamiento y la acción del docente, desde la articulación de la teoría y la práctica, el sentir, pensar y actuar, con un sentido creativo e innovador. También hace mención de la necesidad de atender a los cambios actuales y propone cuatro pilares o bases en los que debe fundamentarse la praxis educativa:

- Aprender a conocer, tener en cuenta los avances de la ciencia y la tecnología y las nuevas tendencias de las actividades sociales y económicas compaginadas con una amplia cultura general.

- Aprender a hacer, adquirir las competencias necesarias que permitan enfrentar disímiles y numerosas situaciones, la mayoría de ellas imprevistas, que faciliten el trabajo en equipo; para lo que propone ejercitar la memoria, la atención y el pensamiento (articulación de lo abstracto con lo concreto), aprendiendo la concentración de la atención en las actividades que ejecuta y en las personas.

- Aprender a ser, explorar todos los talentos que permanecen ocultos en el individuo (memoria, razonamiento, aptitudes físicas, facilidad de comunicación, carisma), con la finalidad de confirmar y comprenderse a sí mismo.

- Aprender a vivir juntos para:

...conociendo mejor a los demás, su historia, sus tradiciones, su espiritualidad, ... crear un espíritu nuevo que impulse la realización de proyectos comunes o a la solución inteligente y pacífica de los inevitables conflictos, gracias justamente a esta comprensión de que las relaciones de interdependencia son cada vez mayores, y a un análisis compartido de los riesgos y retos del futuro... (Delors, 1996 p. 23).

Es significativo precisar que los primeros tres pilares apoyan al último y se complementan para enseñarle al educando a descubrir quién es y por ende conocer al otro, 
colocándose en su lugar, comprendiendo sus reacciones, participando en proyectos cooperativos, deportes, actividades sociales y culturales; entre otros aspectos.

Pero para que todo esto se logre se requiere del consenso de tres agentes: la comunidad local, los padres, directores y los docentes; a fin de establecer ejes y reglamentos concertados y evaluar las necesidades mediante el diálogo con las autoridades públicas y organismos nacionales e internacionales quienes asesoran y prestan el apoyo pertinente.

Este proceso supone que el aprendizaje se debe estar cuestionando permanentemente y en todas las épocas, ya sea durante la crisis o en períodos propicios, para enseñar saberes que permitan cimentar racional y convincentemente la convivencia democrática e introducir cambios positivos que generen y refuercen la capacidad y la confianza en las organizaciones; estas transformaciones deben compartirse con toda la organización, de tal manera que sus miembros puedan aprovecharlas.

En consecuencia las organizaciones que aprenden descubren lo que es efectivo reconstruyendo sus propias experiencias, producto de una construcción colectiva, de una compleja interacción entre los modelos individuales de aprendizaje y el mundo de la conducta organizacional, así como también del proceso indagatorio sobre la detección y corrección de errores basados en la incongruencia entre las teorías declaradas y las teorías en uso, en vista a ajustar objetivos y resultados esperados. En este contexto McGill y Slocum, (1993), afirman que la cultura de aprendizaje tiene características muy particulares.

Entre estas especificidades se destacan la apertura a la experiencia, a fin de estimular la responsable asunción de riesgos y la disposición para reconocer los fracasos y aprender de ellos; por cuanto su efectividad se logra a través del diálogo activo y de las conversaciones armoniosas, pues proporcionan la oportunidad de hablar y pensar juntos sobre los problemas. Se requiere experimentar continuamente para reconocer que el aprendizaje es la única fuente de ventaja estratégica sostenida.

Por otra parte, las características estructurales de las organizaciones que aprenden son: permeabilidad, flexibilidad e intimidad de redes; por lo tanto, la estructura debe considerarse como un proceso interactivo con visión compartida que contiene sistemas de información, los cuales deben ser exactos, oportunos, disponibles para quienes los necesiten y presentados de manera que facilite su uso, a sabiendas de que ello supone capacitar a la gente para comprender la información y su manejo. Asimismo, un sistema de recompensa que reconozca el aprendizaje estimulando el conocimiento, para así aumentar la diversidad intelectual y mejorar la experiencia.
En tal sentido, se requiere de una práctica de recursos humanos que promueva la valoración de las personas no por lo que saben, sino por su capacidad para aprender, detectar y liderar la solución de los problemas, convertidos en líderes capaces de actualizarse, conocer y comprender las más novedosas teorías educativas y gerenciales para asumir enfoques y criterios de acción diferentes a los del pasado, más informados y reflexionados críticamente, para realizar una práctica educativa y administrativa cónsona con los intereses que coadyuven no sólo a mejorar el proceso de enseñanza y aprendizaje, sino a elevar los estándares de calidad de la organización educativa.

En esta intención se requiere considerar que la estructura social condiciona las metas y los propósitos de los docentes, quienes deben identificar estos aspectos, a fin de superar los obstáculos en la consecución de los fines y objetivos deseados. Por ello se concuerda con Carr y Kemmis (1988) cuando afirman que:

La teoría educativa debe orientarse siempre hacia la transformación de las maneras en que los enseñantes se ven a sí mismos y ven su situación, de manera que permita reconocer y eliminar los factores que frustran sus objetivos e intenciones educativas...orientarse a transformar las situaciones que obstaculizan la consecución de las metas educacionales, perpetúan las distorsiones ideológicas e impiden el trabajo racional y crítico. (p.143)

De la misma manera debe proponer nuevas realidades educativas con sentido innovador y democrático, donde prevalezca la convergencia de las miradas y la escucha, para que sean oídos y apreciados los planteamientos y los conocimientos generados por el otro, propiciando el emerger de un tercero generalmente excluido, el nosotros.

Estas tendencias presuponen una nueva orientación del currículo hacia la educación ética y ciudadana, que proponga rupturas con los paradigmas relacionados con problemas de género, étnico, raciales, sociales, económicos, religiosos, culturales, espaciales, entre otros. En atención a ello, Cullen (2004), señala cuatro supuestos como base para un currículo que interprete la demanda social en términos de formación de un ciudadano democrático, participativo y solidario.

Estos supuestos se refieren a fundar racionalmente, en forma codisciplinar, los principios de la ciudadanía, como forma crítica de socialización y de construcción de un ordenamiento social; fundamentar racional y argumentativamente la viabilidad de la participación política y de la responsabilidad social, que especifican la ciudadanía como democrática; enseñar saberes que permitan una reconstrucción democrática crítica del propio poder, para participar, diseminada en la sociedad y en las normas jurídicas que rigen la vida social. Aprender a resolver democráticamente y con argumentación de derechos y 
obligaciones, los conflictos de poder y de normas, para desarrollar una convivencia pluralista, como algo más que la mera tolerancia del diferente.

En ese mismo orden de ideas Pérez Esclarín (1999), fundamentado en Delors y otros quienes enfatizan en que la función esencial de la educación es "conferir a todos los seres humanos la libertad de pensamiento, de juicio, de sentimiento y de imaginación que necesita para desarrollar plenamente sus talentos...Es un viaje interior cuyas etapas corresponden a las de maduración constante de la personalidad” (p.98), propone orientar la educación implícita y explícitamente en valores y garantizar las competencias fundamentales para una sana convivencia. Luego, parafraseando al autor antes citado se refieren ciertos aprendizajes que contribuyen a mejorar la convivencia y sustentan un mejor saber hacer.

a) Aprender a no agredir ni física ni psicológicamente a los compañeros. Por consiguiente hay que aprender a resolver los conflictos mediante la negociación y el diálogo, de manera que todos salgan favorecidos de él; tratando de convertir la agresividad en fuerza efectiva para la creación y cooperación.

b) Aprender a comunicarse, base de la autoafirmación personal y grupal. Los recintos educativos deben enseñar a: conversar, escuchar, expresarse con libertad, aclarar, comprender al otro, defender con firmeza las convicciones sin agredir a quien diciente.

c) Aprender a interactuar con los otros, a tratar con cortesía, a colaborar, a trabajar en equipo, a decidir en grupo y considerar los problemas como retos a resolver.

d) Aprender a cuidarse, a cuidar de los otros y a cuidar el ambiente, los bienes colectivos y públicos porque pertenecen a todos.

e) Aprender a valorar la propia cultura y religión, a ser conscientes de sus raíces y respetar la diversidad cultural y religiosa, combatiendo los dogmatismos, fundamentalismos e intolerancia de quienes quieren imponer una única forma de pensar, de crecer y de vivir, entendiendo que la diversidad y respeto de las minorías es tan importante como el gobierno de las mayorías.

f) Aprender a desarrollar la autonomía personal, la confianza, el respeto, la autoresponsabilidad, la corresponsabilidad, el compromiso personal y social, la cooperación y la solidaridad.
En derivación de lo antes expuesto se concluye que si los demás no tienen condiciones de vida adecuada no será posible la convivencia. Por consiguiente la concepción de estos aprendizajes son factores claves en la constitución de un mejor saber hacer, más informado, fundamentado en reflexiones que devienen de las vivencias en contextos que se conforman en íntimo desarrollo con el devenir del docente como sujeto del conocimiento y de la acción, en instituciones educativas concebidas como espacios para practicar, vivir y desarrollar los valores sociales, personales, organizacionales, entre otros, que se consideran esenciales para el individuo y la colectividad.

Estos procesos se caracterizan por su complejidad y para los que se debe estar responsablemente preparado y ocupado en la formación de comunidades de aprendizaje en torno a tan interesantes temas. Correa de Molina (2004) señala, con respecto a las ideas planteadas, que la gestión del conocimiento en su acepción sistemática, busca dar nuevas orientaciones a problemas de antaño, en relación con la capitalización, creación y difusión de uno de los recursos más valiosos, el conocimiento. La gestión del conocimiento constituye uno de los retos más complejos que debe enfrentar las instituciones educativas, pues ostenta una multiplicidad de momentos, facetas y etapas que se muestran a continuación:

a) Creación de un ambiente cultural adecuado en la institución.

b) Identificación de requerimientos de conocimientos, captura de conocimientos externos y metodologías para la utilización de ellos

c) Articulación gradual de los conocimientos a la cultura organizacional.

d) Dominio de técnicas y metodologías para vislumbrar su importancia en el desarrollo organizacional.

e) Convergencia de varias fuentes de investigación, para el direccionamiento de las tecnologías.

f) Fundamentos teóricos referidos a la teoría de recursos y aprendizaje significativo y organizativo. 
En este orden de ideas, Morin (ob.cit.) plantea la tesis de los desafíos de los desafíos, donde señala que la necesidad de hacer frente a los retos interdependientes, es un problema perentorio de este tiempo. También propone que la reforma del pensamiento permitiría el vínculo de las culturas disociadas.

Por consiguiente la reforma de la enseñanza debe conducir con la del pensamiento pues se trata de una reforma paradigmática, concerniente a la organización y gestión del conocimiento, para navegar certeramente en esta era, es decir, conducir, explorar, manejar y arriesgarse con nuevos modos, más pertinentes a una acción educativa mediadora humanista crítica, como lo explica Ferreiro (1986), cuando se refiere a las etapas del desarrollo de la inteligencia piagetiano, el crecimiento intelectual no consiste en una suma de conocimientos sino en grandes períodos de reestructuración y, en muchos casos, reestructuración de las informaciones anteriores, que cambian la naturaleza al entrar en un nuevo sistema de relaciones.

\section{A MANERA DE CIERRE}

Al docente se le considera un sujeto intelectual y social, también un sujeto orgánico, quien despliega sus acciones mediante mecanismos que lo conducen a trascender el aula, por cuanto da cuenta de un compromiso social que manifiesta no sólo en sus saberes haceres pedagógicos y didácticos, sino en la acción creativa y espontánea en el entorno educativo, a fin de devenir en una educación para la vida, con un fundamento ecológico y humanizante.

Esto se posibilita al conformar una conciencia crítica y madura en la población, para la construcción de una sociedad incluyente y democrática, que pueda organizarse mediante un proyecto, para encontrar soluciones vinculadas a la vez, tanto con los problemas de integración mundial, como con los problemas locales, con el fin de aproximarse a una situación global y nueva, donde se estimen las visiones colectivas en pro de la universidad y la sociedad.

Para ello requiere articular lo científico, lo filosófico y lo estético, a través de una acción poderosa, como lo es la interformación docente, realizada en forma responsable, democrática, participativa y colaborativa, instituyéndose como un auténtico pedagogo, constructor de su saber disciplinar, pedagógico y didáctico, con un discurso autoconstituido, potenciador del capital intelectual, social y académico de la universidad, así como de la naturaleza creativa-participativa-afectiva de los estudiantes.

\section{REFERENCIAS}

Bachelard, G. (1985). La Formación del Espíritu Científico. México: Siglo XXI.

Barabtarlo, A. "El Docente Intelectual Orgánico: su proceso de socialización”. 1998. [Documento en línea]. Disponible: me.//c:WINDOWS.Escritorio. Barba.htm. [Consulta: 2000, Julio 25].

Barbero, J. (2006). La Razón Técnica Desafía a la Razón Escolar. Bogotá: Cooperativa Editorial Magisterio

Carr, W. y Kemmis, S. (1988). Teoría Crítica de la Enseñanza. Barcelona: Martínez

Correa de Molina, C. (2004). Gestión y evaluación de calidad. Referentes para la acreditación. Bogotá: Cooperativa Editorial Magisterio

Cullen, C. (2004) Autonomía moral, participación democrática y cuidado del otro. México: Ediciones Novedades Educativas.

Delors, J. (1996). La Educación Encierra un Tesoro. Informe. Madrid: Santillana. Ediciones UNESCO.

Ferreiro, E. (1986). Procesos de alfabetización. La Alfabetización en Proceso. Buenos Aires: Centro Editor de América Latina.

Giroux, H. (1990). Teoría y Resistencia en Educación. Una pedagogía para la oposición. México: Siglo XXI editores.

González, F. y Mitjáns, A. (1989). La Personalidad su Educación y Desarrollo. La habana: Editorial pueblo y educación.

Habermas, J. (1986). El Discurso Filosófico de la Modernidad. Madrid: Editorial Taurus.

McGill, M. y Slocum, J. (1993). Unlearning the Organization. Organizational Dynamics. Vol. 22, No. 2. Material Mimeografiado. Resumen - Traducción: Gordana Samek de Rodríguez.

Manterola, C. (s/f). ¿Qué Pensamiento Epistemológico usas en tu Clase?. Aquí entre Nosotros (183) pp. 41-53. 
Morin (1999). Los Siete Saberes Necesarios a la Educación del Futuro. Caracas: Ediciones FACES / UCV.

Pérez-Esclarín, A. (1999). Educar en el Tercer Milenio. Caracas: Editorial San Pablo.

Porlán, R. y Rivero, A. (1998). El Conocimiento de los Profesores. Sevilla: Década Editores S.L.

Schön, D. (1992). La Formación de Profesionales Reflexivos. Hacia un nuevo diseño de la enseñanza y el aprendizaje en las profesiones. Barcelona: Paidós.

Sinclair, H. (1981). Recientes investigaciones piagetianas sobre los modelos de aprendizaje. Buenos Aires: Editorial Huemul.

Tünnermam, B. (1999). "La Declaración Mundial sobre la Educación Superior en el siglo XXI: Una lectura desde América Latina y el Caribe”. Educación Superior y Sociedad. 10. (1), 7-34. 